AperTO - Archivio Istituzionale Open Access dell'Università di Torino

\title{
Mechanochemical and sonochemical heterocyclizations
}

\section{This is the author's manuscript}

Original Citation:

Availability:

This version is available http://hdl.handle.net/2318/1637319

since 2018-02-21T18:10:08Z

Published version:

DOI:10.1007/s10593-017-1979-y

Terms of use:

Open Access

Anyone can freely access the full text of works made available as "Open Access". Works made available under a Creative Commons license can be used according to the terms and conditions of said license. Use of all other works requires consent of the right holder (author or publisher) if not exempted from copyright protection by the applicable law. 
This is the author's final version of the contribution published as:

Gaudino, Emanuela Calcio; Tagliapietra, Silvia; Mantegna, Stefano; Cravotto, Giancarlo. Mechanochemical and sonochemical heterocyclizations.

CHEMISTRY OF HETEROCYCLIC COMPOUNDS. 52 (11) pp: 1-10. DOI: $10.1007 / \mathrm{s} 10593-017-1979-\mathrm{y}$

The publisher's version is available at:

http://link.springer.com/10.1007/s10593-017-1979-y

When citing, please refer to the published version.

Link to this full text:

http://hdl.handle.net/2318/1637319 


\title{
Mechanochemical and sonochemical heterocyclizations
}

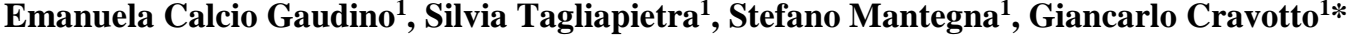 \\ ${ }^{1}$ Dipartimento di Scienza e Tecnologia del Farmaco and NIS-Centre \\ for Nanostructured Interfaces and Surfaces, University of Turin, \\ Via P. Giuria 9, 10125 Turin, Italy; e-mail: giancarlo.cravotto@unito.it
}

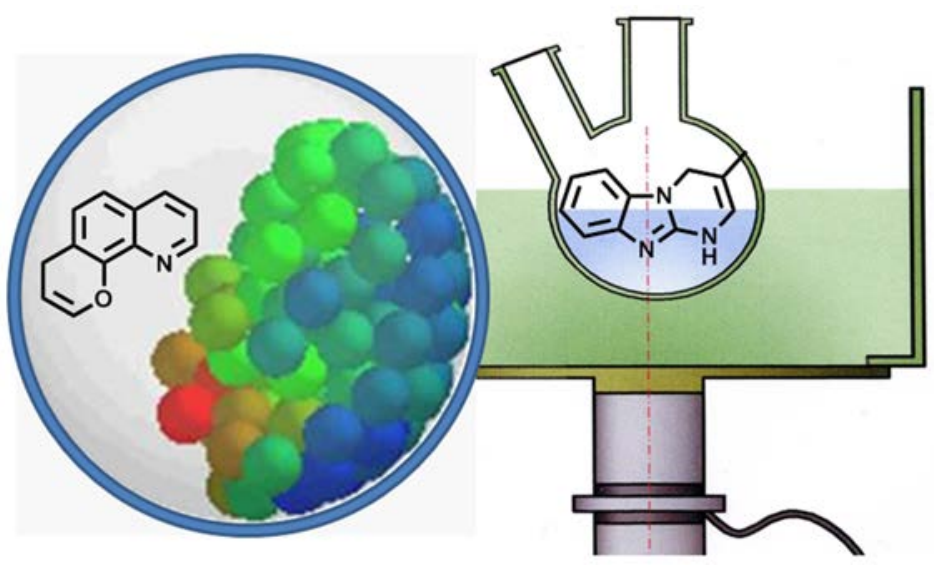

Heterocycle formation reactions require efficient catalysis and suitable physical activation. Sonochemistry and mechanochemistry can dramatically promote heterocyclizations in heterogeneous conditions using benign solvents and even in solventless conditions by means of ball mills. Besides facilitating quick cyclization, these technologies bring with them safer protocols, cost reduction, energy saving, process intensification, and waste minimization. The aim of this review is to describe a number of relevant synthetic advances in the last two years and to highlight the huge unexploited potential of both sonochemistry and mechanochemistry.

Keywords: heterocycles, ball mill, cyclization, green chemistry, ultrasound.

The environmental impact of chemical manufacturing affects both humans and ecological resources. The "green chemistry" revolution and the presence of enabling technologies are providing chemists with an enormous number of organic synthesis challenges, as they search for clean processes. The development of simple, cost-effective, and ecofriendly procedures for the synthesis of heterocyclic compounds was the driving force behind the discovery of green activation methods and non-conventional energy sources. ${ }^{1}$ In fact, sonochemistry and mechanochemistry are capable of creating unique high-energy microenvironments that strongly promote original synthetic pathways. Ultrasound (US) and mechanical action have shown enormous advantages when compared to conventional heating and stirring in heterogeneous systems, improving reaction rates, yields, and even selectivity. High-intensity US induces strong acoustic cavitation and streaming which enable efficient mixing, catalyst surface activation, and particle dispersion to occur. ${ }^{2}$ Meanwhile, acoustic cavitation and grinding may well address the criteria of sustainability in terms of cost, energy consumption, and waste minimization. ${ }^{3}$ This review will discuss both on-going and future directions in the interplay of sonochemistry and mechanochemistry in heterocyclization.

\section{Cyclization under mechanochemical activation}

Thermal and inverse heating using microwaves are the two most common techniques for the controllable accomplishment of chemical reactions. Apart from radiation-based mechanisms, other methods of delivering energy are: electricity (electrochemistry), cavitation processes (acoustic, hydrodynamic) and mechanical energy (friction, impact, collision). According to the IUPAC definition, ${ }^{4}$ a mechanochemical reaction is defined as "a chemical reaction that is induced by the direct absorption of mechanical energy". However, mechanochemistry is much more than just the reactive treatment of substances. The field is further divided into (i) the mechanical activation of solids, (ii) mechanical alloying, and (iii) the reactive milling of solids. Particle refinement due to comminution leads to an increase in surface area, surface energy, and number of defects. Increased surface energy can result in mechanochemical activation if alterations in structure, chemical composition, and/or chemical reactivity occur during the milling.

In 2011, Stolle et al. ${ }^{5}$ published a review with the main objective of motivating researchers to consider ball mills as a versatile technique and a useful reactor for extensive use in synthetic labs. Heterocycle syntheses typically include a condensation step, and there is usually no way to remove the freed water from closed ball mill vessels during the grinding. Nevertheless, the literature holds many examples of this type of synthesis taking advantage of the thermodynamic stability of the formed products. In 1999, Kaupp et al. ${ }^{6}$ were the first to investigate pyrrole and indole syntheses using the ball- 
milling technique. They reported the one-pot reaction of trans-1,2-dibenzoylethene with primary or secondary enamine esters or enamine ketones using ball milling and without any liquid phase. The reaction entails the Michael addition of the enamine nitrogen, followed by the cycloaddition of the enamine double bond, a rearrangement to enamine and the final elimination of water. The corresponding pyrrole (1) or indole (2) products were obtained quantitatively (Scheme 1).

\section{Scheme 1}

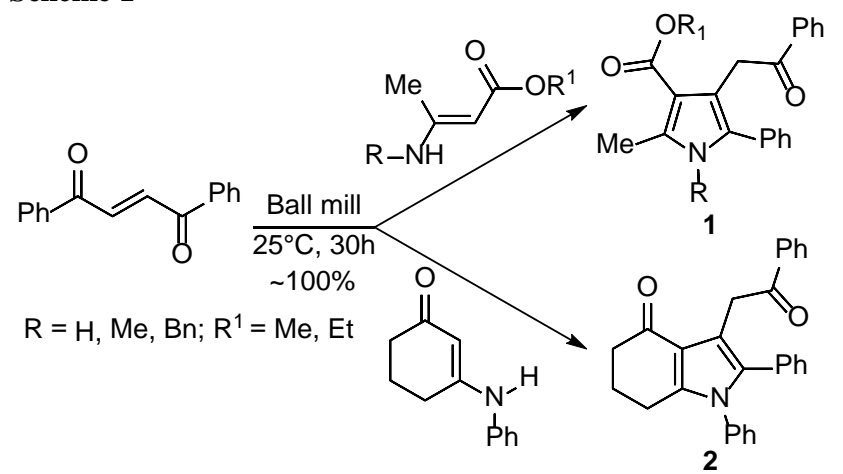

In a recent review, M'hamed has shown that mechanochemically assisted heterocycle synthesis is one of the most promising "green" methods available and one that provides several advantages over conventional procedures. ${ }^{7}$ This trend has seen confirmation over the last year with the exponential growth of new examples. For example, Akelis et al. ${ }^{8}$ have reported on a fast and easy method for the preparation of $N$-substituted pyrroles 3 via mechanical activation by ball milling, using solid bio-sourced "green acids", such as citric acid, pyroglutamic acid, succinic acid, ascorbic acid, camphorsulfonic acid, and oxalic acid (Scheme 2). The effectiveness of the acid catalysts under mechanochemical conditions was assessed using blank tests where amine and diketone were shaken at $30 \mathrm{~Hz}$ for 15 or $30 \mathrm{~min}$ without any acid catalyst. The presence of the acid catalyst is required to form pyrrole from amine and diketone. Compared to previously reported methods, the main advantages of the present procedure lie in the absence of a solvent and the use of a "green" organic acid that is nontoxic and produced from biomass.

\section{Scheme 2}

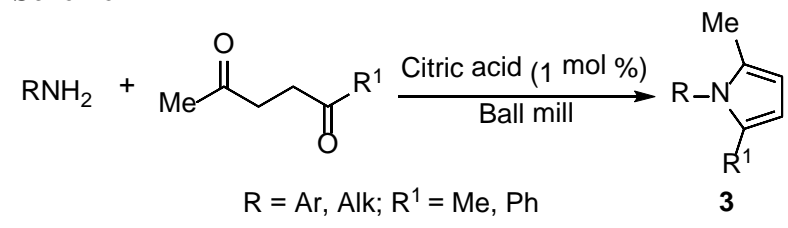

Miklós et al. ${ }^{9}$ have prepared a series of new N-heterocyclic spiroadamantanes 4 through the reaction of 2-adamantanone with $\beta$-aminocarboxamides and $\beta$-aminocarbohydrazides under ball-milling (Scheme 3). Almost quantitative yields (99\%) were obtained after $4 \mathrm{~h}$ of grinding. The use of $\mathrm{ZrO}_{2}$ balls instead of stainless steel balls was essential to avoiding paramagnetic impurity effects in the NMR determination of the crude product conversions.

\section{Scheme 3}

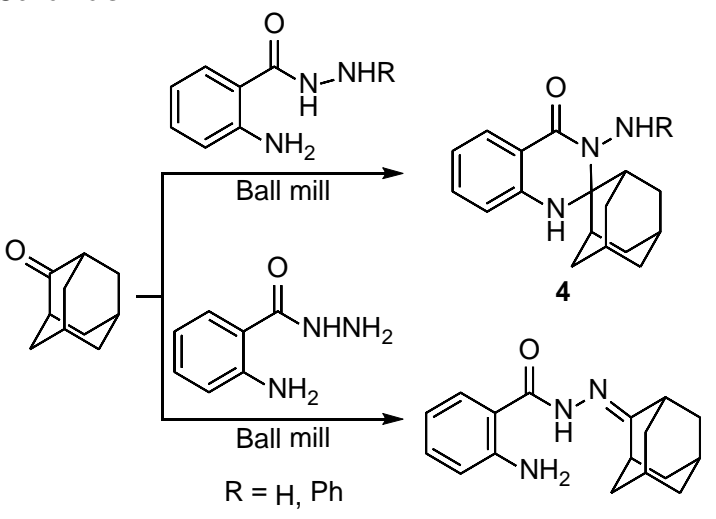

An effective method for the synthesis of 2-aryl-1-arylmethyl-1H-benzimidazoles 5 has been described by Jin et al.: ${ }^{10}$ a solventless condensation of $o$-phenylenediamine with aldehydes in a ball mill, using $\mathrm{FeCl}_{3} \cdot 6 \mathrm{H}_{2} \mathrm{O}$ as a catalyst (Scheme 4). The selective formation of 1,2-disubstitued benzimidazole 5, without detectable levels of 2-monosubstituted benzimidazoles being produced, occurs regardless of the amine/aldehyde molar ratio. Moreover, imine and bis-imine are formed quantitatively from the condensation of $o$-phenylenediamine with one or two equivalents of aldehyde in a ball mill without Fe(III). The role of the catalyst in the mechanochemical cyclization and aromatization to benzimidazole derivatives was crucial. On the other hand, cyclizations are facilitated by subsequent oxidation steps in conventional solution preparations. This further indicates that the reaction pathway and selectivity can change in the presence of a Fe(III) catalyst 
in a ball mill. This is a versatile method which is effective on a wide range of substrates under mild reaction conditions, providing easy workup where pure products are recovered via simple filtration and crystallization.

\section{Scheme 4}

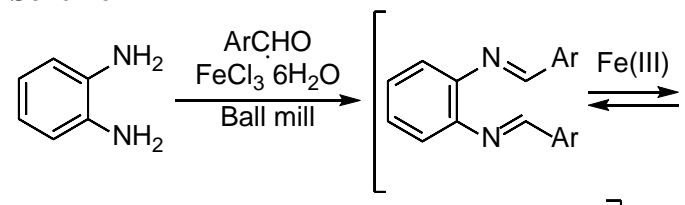

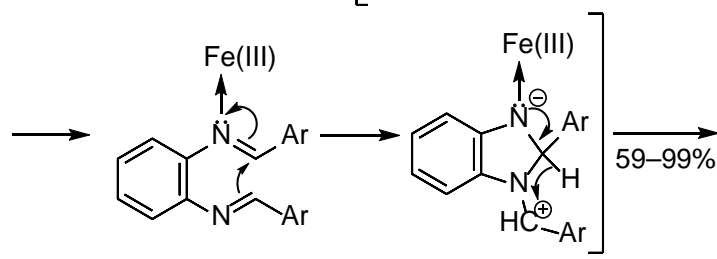<smiles>CCn1c(Br)nc2ccccc21</smiles><smiles>Cc1ccc2nc(Br)[nH]c2c1</smiles>

An efficient mechanochemical route to 2-arylbenzothiazoles 6 and both 2-substituted and 1,2-disubstituted benzimidazole derivatives using simple agate mortar-pestle grinding has been described by Banerjee et al. ${ }^{11}$ (Scheme 5 ). In the liquid-assisted grinding method, a little amount of solvent can partly dissolve solid intermediates and thereby release trapped starting material to facilitate complete conversion. It is well-documented that the liquid-assisted grinding often brings out better results than "dry" grinding. 2-Substituted benzothiazoles 6 were synthesized in high yields and short reaction times (10-60 min) from $o$-aminothiophenol and a variety of aromatic aldehydes (1.2 equiv) in a simple mortarpestle grinding in the presence of EtOH at room temperature. From a mechanistic point of view, the reaction starts with the spontaneous formation of a Schiff base, followed by the nucleophilic attack of a sulfur atom on the imine, forming the 1,2dihydrobenzothiazole derivative which is then oxidized to 2-phenylbenzothiazole by atmospheric oxygen in the ball mill.

Scheme 5

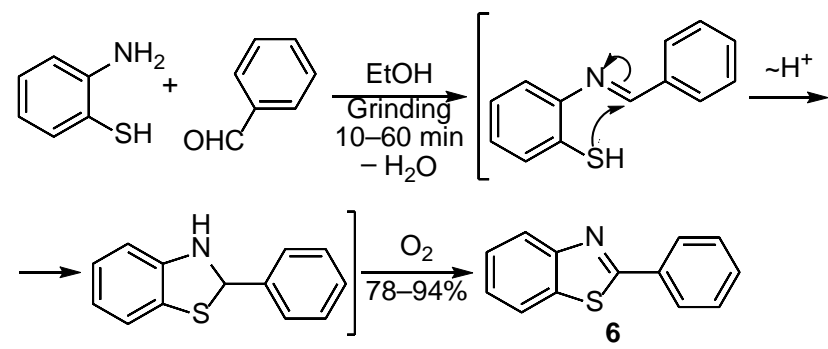

In 2006, Tulleberg and coauthors ${ }^{12}$ described the Heck-Jeffery reaction of various haloanilines with an amido acrylate under solvent- and phosphine-free conditions and the subsequent cyclization of suitably substituted coupling products 7 to their corresponding indole derivatives 8 (Scheme 6). The reaction either failed or gave low yields (29-31\%) with iodoanilines bearing electron-withdrawing groups $\left(\mathrm{CN}, \mathrm{CO}_{2} \mathrm{CH}_{3}, \mathrm{CF}_{3}\right)$. Yields were also influenced by the position of the amino or hydroxyl substituent with respect to the iodine atom. Results were better when the heteroatom was in either the meta or para positions $\left(\mathrm{R}^{2}\right.$ or $\mathrm{R}^{3}=\mathrm{NH}_{2}$ or $\mathrm{OH}$, yields $\left.64-85 \%\right)$ and poor to average when it was in the ortho position $\left(\mathrm{R}^{1}=\right.$ $\mathrm{NH}_{2}$ or $\mathrm{OH}$, yields 29-43\%). This effect can, to some extent, be explained by the increase in steric hindrance at the iodine atom, but it may also be an electronic effect caused by the coordination of nitrogen to palladium. The presence of nitrogen influences the electronic properties of Pd(II) in the oxidative addition complex. This, in turn, may slow down or completely stop subsequent steps in the Heck reaction. Nevertheless, 2-haloaniline substrates gave Heck coupling products, but some of the primary coupling products easily cyclized to the indole derivatives. 
Scheme 6

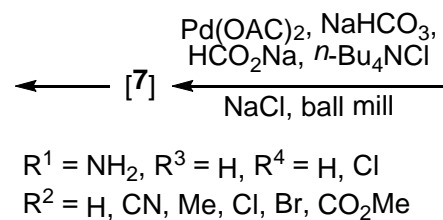

$\mathrm{R}^{2}=\mathrm{H}, \mathrm{CN}, \mathrm{Me}, \mathrm{Cl}, \mathrm{Br}, \mathrm{CO}_{2} \mathrm{Me}$

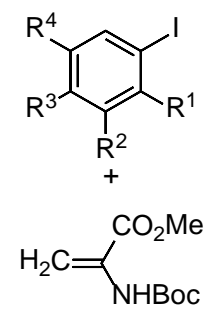
$\mathrm{Pd}(\mathrm{OAC})_{2}, \mathrm{NaHCO}_{3}$ $\mathrm{HCO}_{2} \mathrm{Na}, n-\mathrm{Bu}_{4} \mathrm{NCl}$
$\mathrm{NaCl}$, ball mill

$\mathrm{R}^{1}=\mathrm{NH}_{2}, \mathrm{H}, \mathrm{Me}, \mathrm{OH}$<smiles>[R]c1cc(/C=C(\NC(=O)OC)C(=O)OC)c([R])c([R])c1[R]</smiles>

$\mathrm{R}^{2}=\mathrm{H}, \mathrm{NH}_{2}, \mathrm{Me}$

$\mathrm{R}^{3}=\mathrm{NH}_{2}, \mathrm{H}, \mathrm{CF}_{3}, \mathrm{OH}$

$\mathrm{R}^{4}=\mathrm{CN}, \mathrm{CO}_{2} \mathrm{Me}, \mathrm{H}, \mathrm{NH}_{2}, \mathrm{~F}$

Shy et al. ${ }^{13}$ have presented a mechanochemical synthesis of tetraphenylporphyrin (TPP) 9 that proceeds via the grinding of equimolar amounts of benzaldehyde and pyrrole in the presence of an acid catalyst to give a solid pink powder by cyclization (Scheme 7). This powder can then be oxidized either in air or in chloroform with an organic oxidizer or, in a second grinding step, with an oxidizing agent to afford TPP. This synthesis was also applied to substituted benzaldehydes to obtain a variety of meso-tetrasubstituted porphyrins (yields 3-33\%). Since the cyclocondensation reaction between an aldehyde and pyrrole is reversible, it was hypothesized that leaving the ground pink solid to stand for several days, while it underwent oxidation in air, allows the cyclized product to revert to the starting material before being oxidized to the porphyrin, resulting in low yields. The second step was a mechanochemical oxidation with various solid oxidizing agents, such as sodium perborate, sodium ethoxide, and oxone that generally gave low yields. The milling time for the oxidation step was varied from 20 min to $8 \mathrm{~h}$. In some cases, a grinding agent (sodium chloride, silica, and magnesium sulphate) was added to the reaction to aid with the grinding process by reducing stickiness, increasing friction and/or removing water from the reaction.

\section{Scheme 7}

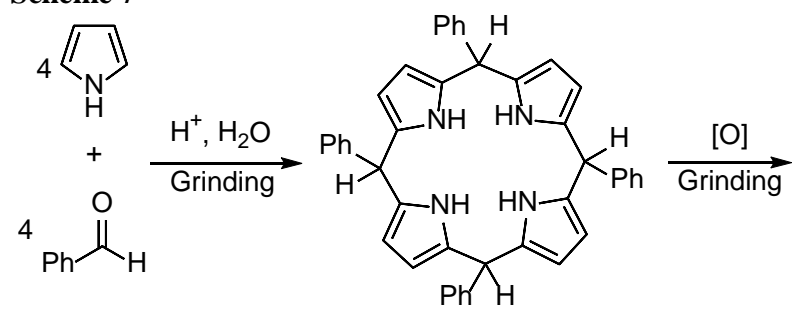<smiles></smiles>

In a continuation of the previous work on intermolecular hydroamination reactions in ball mills, Stolle et al. in $2015^{14}$ have described the reaction of various ortho-substituted anilines with electron-deficient alkynes in a ball mill. The reaction with diethyl acetylenedicarboxylate in a planetary ball mill enabled the formation of the corresponding $N$-vinyl derivatives to occur via intermolecular hydroamination (yield 20-95\%). The $N$-vinyl derivative subsequently underwent amidation/lactonization to the annulated cycle. Benzoxazines 10, quinoxalines 11, and benzothiazines 12 have been synthesized from $o$-hydroxyanilines, $o$-phenylenediamine, and $o$-aminothiophenol, respectively, using this sequential reaction procedure (Scheme 8). The comminution of $o$-aminophenol with diethyl acetylenedicarboxylate in a planetary ball mill with $\mathrm{NaCl}$ alone yielded benzoxazine $\mathbf{1 0}$ (yield $>95 \%$ ) rather than the corresponding $N$-vinylated product. 


\section{Scheme 8}

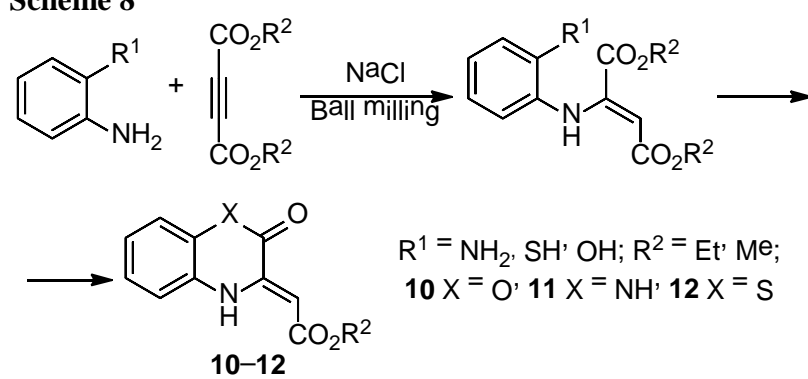

The synthesis of N,N-bicyclic pyrazolidinone derivatives $\mathbf{1 3}$ through the 1,3-dipolar cycloaddition reaction of azomethine imines with terminal alkynes using a vibratory ball mill in the presence of calcium fluoride and copper(I) salt has been described by Chen et al. ${ }^{15}$ (Scheme 9$)$. In a typical procedure, a mixture of azomethine imine $(0.40 \mathrm{mmol})$, ethyl propiolate $(0.40 \mathrm{mmol})$, $\mathrm{CuI}(0.04 \mathrm{mmol})$, and $\mathrm{SiO}_{2}(500 \mathrm{mg})$ was vigorously milled at a rate of $20 \mathrm{~Hz}$ at room temperature for $60 \mathrm{~min}$. Silica is a good grinding aid. To avoid overheating, the reactions were often carried out in cycles consisting of a $10 \mathrm{~min}$ milling period followed by a 5 min pause at room temperature. The final product, after purification, was obtained in a $40 \%$ yield. Of the several additives tested (amines, tetrabuthylammonium fluoride, potassium fluoride, etc.) calcium fluoride was the best, as it gave $80 \%$ yield (molar ratio azomethine imine : ethyl propiolate : $\mathrm{CuI}: \mathrm{CaF}_{2}=1.2: 1: 0.1: 0.2$ ). In some cases, CuOAc was a better catalyst than CuI, giving higher yields without any detectable homocoupling by-products. Copper acetylide is probably produced from cuprous acetate in the presence of $\mathrm{CaF}_{2}$, and the displaced hydrogen would then combine with acetate to provide an acidic environment. The copper acetylide would then interact with the azomethine imine and rearrange to a putative six-membered metallacycle, which could evolve to a bicyclic vinyl copper species, then protonated in acidic conditions to afford the adduct.

\section{Scheme 9}<smiles>C#CC(=O)OCC</smiles>

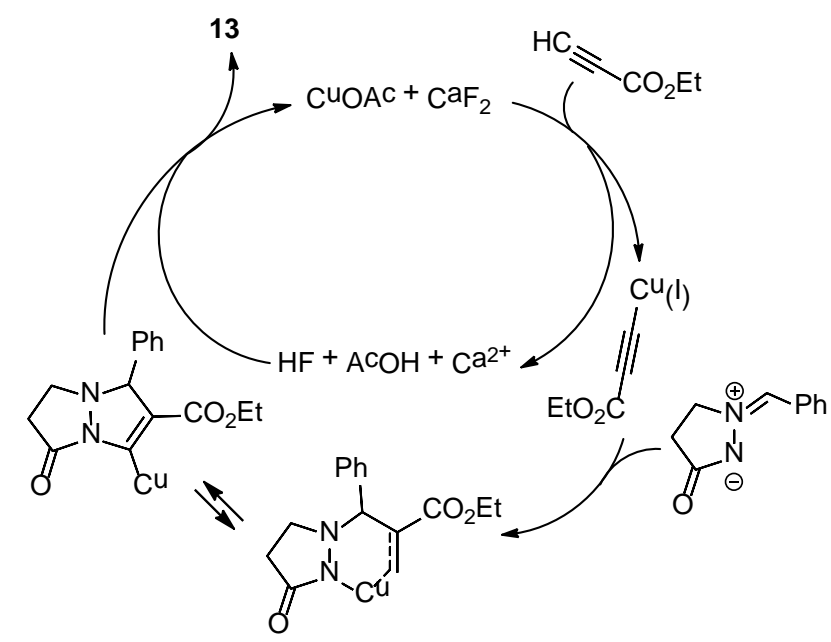

\section{Cyclization under ultrasound}

In the last few decades "sonochemistry" (or "cavitational chemistry") was one of the most important techniques in organic synthesis and green chemistry. As hereinafter described, a large number of heterocycle formation reactions have been efficiently performed under mild conditions with ultrasonic irradiation.

Wang et al. ${ }^{16}$ were the first to demonstrate that lactic acid was an effective natural compound-based green solvent medium for scalable Knorr condensation reactions under US irradiation. The sonochemical reaction afforded the corresponding pyrrole products 14 in only $30 \mathrm{~min}$ at $60^{\circ} \mathrm{C}$ in $75-95 \%$ yield (Scheme 10). The present method under US avoids the harsh reagents and organic solvents of the traditional Knorr condensation in acetic acid. The solvent is replaced by the inexpensive and safe lactic acid, which is well suited for industrial production. 


\section{Scheme 10}
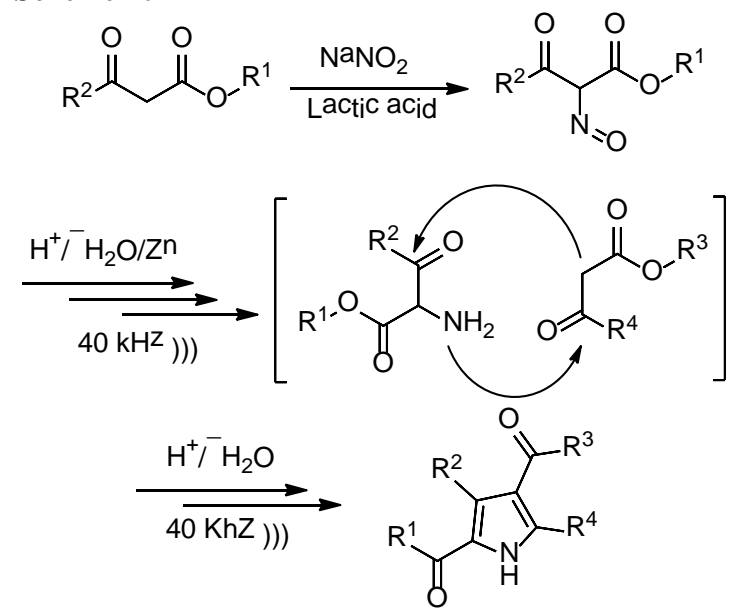

14

Pyrrolidin-2-one and $\gamma$-lactam derivatives have attracted a great deal of attention over the past decade thanks to the wellestablished bioactive effects of piracetam-like compounds. In this context, Franco et al. ${ }^{17}$ have explored the viability of using US irradiation to promote the reaction of methyl 7,7,7-trifluoro-4-methoxy-6-oxohept-4-enoate with primary alkyl(aryl)amines to prepare 5-(3,3,3-trifluoro-2-oxopropylidene)pyrrolidin-2-one derivatives $\mathbf{1 5}$ (Scheme 11). The simple one-pot sonochemical reaction gave high yields (80-95\%) in shorter reaction times (120 min) at $55^{\circ} \mathrm{C}$.

\section{Scheme 11}

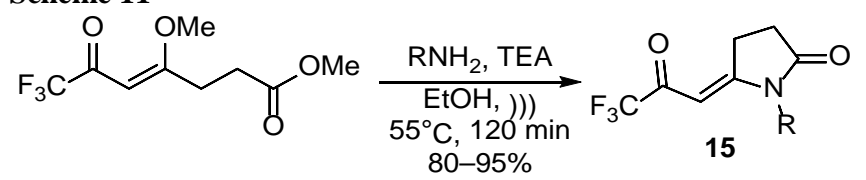

Recent years have seen a large number of pyrazole derivatives synthesized and tested. ${ }^{18-22}$ In this context, Nemati et al. ${ }^{23}$, have reported the cost-effective, eco-friendly one-pot synthesis of highly substituted pyrazole derivatives 16a,b by reacting various aldehydes, malononitrile, and either phenylhydrazine or 4-phenylthiosemicarbazide in PEG-400 and water at room temperature under US irradiation (Scheme 12).

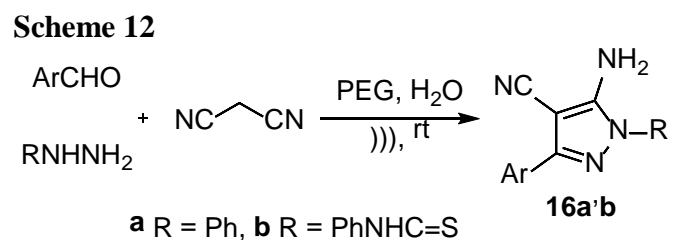

Multicomponent reactions (MCRs) are gaining importance and are in high demand in modern organic synthesis. This is particularly true in the case of heterocycles. ${ }^{24-25}$ Shabalala et al. ${ }^{26}$ have reported MCRs that provide easy access to clean synthetic routes for heterocyclic derivatives and a US-assisted method for the synthesis of polysubstituted pyridines. The same authors analogously prepared pyrazole derivatives under US, using water as a solvent. In that pursuit, they have reported the one-pot synthesis of tetrahydropyrazolopyridine derivatives (dipyrazolo[3,4-b:4',3'-e]pyridines) 17 and pyrano[2,3-c]pyrazoles 18 via four-component coupling reactions under US irradiation at $50^{\circ} \mathrm{C}$ in water within $0.5-2.5 \mathrm{~h}$. The fast US-assisted MCR gave good to excellent yields (80-90\%) (Scheme 13). 


\section{Scheme 13}<smiles>[R]c1ccc(C2C(C#N)=C(N)Oc3[nH]nc(C)c3C2c2ccc([Y6]([H])([H])[H])cc2)cc1</smiles>

The 1,2,3-triazole unit has received great interest and special attention because it appears in the structures of compounds suitable for medicinal application. Mady et al. ${ }^{27}$ have described the US-assisted click reaction to access a series of $1,2,3-$ triazoles linked to an diaryl sulfone moiety 19 via copper-catalyzed azide-alkyne 1,3-dipolar cycloaddition (CuAAC) in aqueous tert-butanol (Scheme 14). The in situ one-pot formation of azides from $\alpha$-bromo ketones followed by the CuAAC enabled the preparation of a library of compounds (yield 90-95\% in 30-60 min).

\section{Scheme 14}<smiles>[X]c1ccc(C(=O)CBr)cc1</smiles>

A number of different 1,2,3-triazole-based benzothiazoles 20 have been designed and synthesized from the corresponding 2-aminobenzothiazoles by Rezki et al. ${ }^{28}$ (Scheme 15). The US-assisted synthetic approach (50 kHz, $240 \mathrm{~W}$ ) consisted of CuAAC between the appropriate 2-azido- $N$-(1,3-benzothiazol-2-yl)acetamides with different terminal alkynes to regioselectively furnish novel 1,2,3-triazoles with a benzothiazole moiety tethered. Significant reductions in reaction times (4 h vs $10 \mathrm{~h}$ ) and higher yields (>95\% vs 80\%) were observed when the reactions were carried out under US irradiation.

\section{Scheme 15}<smiles>[R17]c1ccc2nc(NC(=O)CN)sc2c1</smiles><smiles>[R16]c1ccc2nc(NC(=O)Cn3cc([R])cn3)sc2c1</smiles>

US irradiation has also been applied by Naeimi and Kiani ${ }^{29}$ to the fast synthesis of 1-substituted tetrazoles 21 through a cyclization reaction between various primary amines, sodium azide, and triethyl orthoformate (Scheme 16). This reaction was effectively catalyzed by ZnS nanoparticles, which worked as an efficient, recoverable, and reusable catalyst. Compared with conventional methods, US gave shorter reaction times, an easier work-up, and higher yields under milder conditions. The effects that solvents, such as acetonitrile, ethanol, DMF, and water, have on the reaction were also investigated. The best yields (92\%) were achieved in only $20 \mathrm{~min}$ US irradiation at $50 \mathrm{~W}$ in DMF. 


\section{Scheme 16}

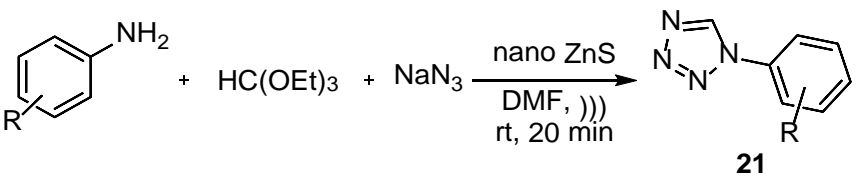

Carradori et al. $^{30}$ have developed an alternative microwave- and US-assisted procedure both for the synthesis of thiosemicarbazone and the subsequent thiazole ring closure (Hantzsch cyclization). A series of 4,5-substituted (thiazol-2yl)hydrazine derivatives 22 were synthesized under sonication $(40 \mathrm{kHz})$ at $50^{\circ} \mathrm{C}$ by reacting 1 -(propan-2ylidene)thiosemicarbazide with variously functionalized halo ketones (Scheme 17). Short reaction times, easy work-up, and the environmentally friendly nature of the synthetic pathway make this method of improving the known heterocyclic chemistry.

\section{Scheme 17}

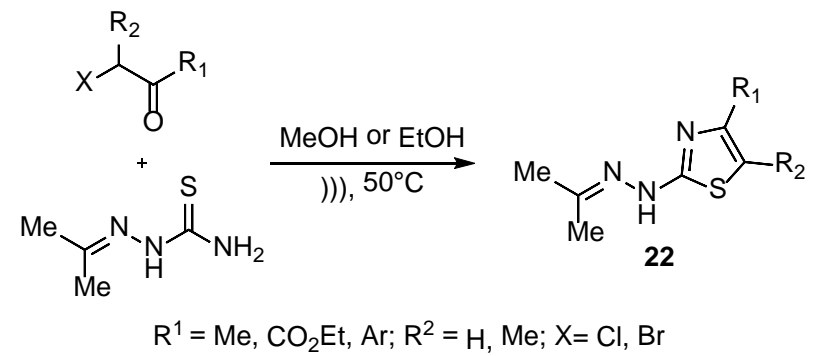

A US-promoted, one-pot MCR for the synthesis of 2-\{2-[(3-aryl-1-phenylpyrazol-4yl)]methylidene \}hydrazinylidenethiazolidin-4-one derivatives 23 has been carried out in an 20 mol \% aqueous emulsion of cetyltrimethylammonium bromide $(\mathrm{CTAB})$ at $60^{\circ} \mathrm{C}$, giving good to excellent yields. ${ }^{31}$ In particular, freshly prepared substrates, 3-(4-aryl)-1-phenyl-1H-pyrazole-4-carbaldehydes, have been condensed with thiosemicarbazide and ethyl bromoacetate in an aqueous emulsion of CTAB (Scheme 18). This US-assisted method offers several advantages, such as operational simplicity, ease of work-up, shorter reaction times, and high yields of compounds 23.<smiles>[R]c1ccc(-c2cc([R])ccc2-c2nn(-c3ccccc3)cc2/C=N\N=C2/NC(=O)CS2)cc1</smiles>

Darehkordi et al. ${ }^{32}$ have developed a simple synthetic method for the preparation of ethyl 2-(2-alkoxy-2-oxoethylidene)5-aryl-7-methyl-3-oxo-2,3-dihydro-5H-thiazolo[3,2-a]pyrimidine-6-carboxylate derivatives 24 under US irradiation (45 $\mathrm{kHz}$ ) in methanol at room temperature (Scheme 19). US promoted the reaction of pyrimidinone derivatives with dimethyl acetylenedicarboxylate to give the corresponding adduct $\mathbf{2 4}$ (yield 95\% in only 25 min, vs 2 h under magnetic stirring). 


\section{Scheme 19}<smiles>CCOC(=O)C1=C(C)NC(=S)NC1[Al]</smiles><smiles>[Z4]N=C1S/C(=C/C(=O)OC)C(=O)N1C1N=C(C)C(C(=O)OCC)=C1C</smiles>

A new class of anticancer derivatives with benzo[5,6]isoindolo[1,2- $a]$ phthalazine and benzo[f]pyridazino[6,1- $a]$ isoindole skeletons have been prepared via heterocyclic cyclization under US irradiation by Mantu et al. (2014) ${ }^{33}$. The polycyclic 1,2diazines 25a,b were efficiently (yields of 60\% in a few minutes) prepared under US irradiation in two synthetic steps; the $\mathrm{N}$-alkylation of either the pyridazine or phthalazine heterocycle, followed by a [3+2]-dipolar cycloaddition of the resulting 1,2-diazinium ylides to the corresponding dipolarophiles (Scheme 20).

\section{Scheme 20}<smiles>[Z]C[Y8]1cc2ccccc2cn1</smiles>

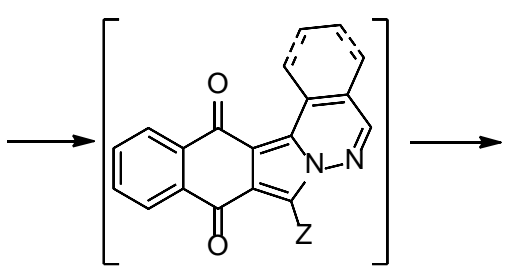<smiles></smiles>

$25 a$<smiles>[Z]c1c2c(c3cccnn13)C(=O)c1ccccc1C2=O</smiles>

25b

$\mathrm{Z}=\mathrm{CONH}_{2}, \mathrm{CO}_{2} \mathrm{Me}, \mathrm{CO}_{2} \mathrm{Et}$

MCRs are well suited to sonochemical conditions where their typical advantages (shorter reaction times, process simplification, etc.) are maximized. This approach has also been widely exploited in medicinal chemistry to provide libraries of biologically active compounds, including heterocycles. Gouvêa et al. ${ }^{34}$ have reported the US-assisted synthesis of thiazinan-4-one derivatives $\mathbf{2 6}$ via the MCR of 2-morpholinoehtylamine, aromatic aldehydes; and 3-mercaptopropionic acid (Scheme 21). The thiazinanones were obtained in 41-88\% yield in only 25 min using a US probe (20 kHz).

\section{Scheme 21}<smiles>NCCN1CCOCC1</smiles>

The efficient synthesis of a novel series of 12-substituted 2-amino-3-cyano-4H-pyran derivatives 27 by means of a onepot three-component cyclocondensation reaction of heteroaryl aldehydes, malononitrile, and active methylene compounds, which is catalyzed by iodine in aqueous medium under US irradiation, has been reported by Tabassum et al. ${ }^{35}$ This protocol (Scheme 22) offers several advantages over more conventional methods, such as shorter reaction times, higher yields, milder conditions, and environmental friendliness, thus successfully demonstrating the synergy between MCR and sonication. The mechanism suggested for product formation involves sequential conversions starting with the Knoevenagel condensation of malononitrile with aldehyde to a heterylidenenitrile derivative. This particular process may just take place 
with a catalytic amount of iodine which increases the electrophilic character of the aldehyde and activates the carbonyl group toward the nucleophilic attack of malononitrile. A Michael addition subsequently takes place to give the intermediate. The nitrile group is then attacked by the enolate oxygen (Thorpe-Ziegler type reaction) followed by a tautomeric proton shift to give the target compounds 27 in $85-97 \%$ yields in only 10 min sonication at $35 \mathrm{kHz}, 80 \mathrm{~W}$, and $25^{\circ} \mathrm{C}$.

\section{Scheme 22}

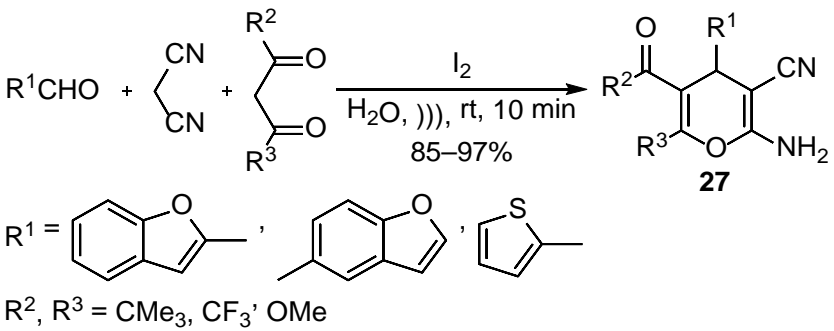

This method should increase the structural diversity within the 2-amino-3-cyano-4H-pyran family. It is envisaged that the sonochemical conditions present in solvent water will help the protocol develop further and provide good prospects for industrial applications, synthetic chemistry and materials science.

The multicomponent condensation of 8-hydroxyquinoline, aromatic aldehydes, and sulfones catalyzed by $p$ toluenesulfonic acid for the synthesis of $4 H$-pyrano[3,2- $h$ ]quinoline derivatives $\mathbf{2 8}$ has been reported by Al-Bogami et al. ${ }^{36}$ The sonochemical MCR was carried out in ethanol (Scheme 23). It is presumed that the reaction starts with a Knoevenagel reaction in the presence of $p-\mathrm{TsOH}$ as a catalyst via the condensation of protonated aldehydes and sulfone derivatives. A Michael addition with 8-hydroxyquinoline, followed by cyclization and rearrangement, then provides the target compounds 28. The simple one-pot nature of the reaction makes it an interesting alternative to other multistep approaches. Sonochemical protocols gave an improvement in rates (45 min vs 8 h) and yields (85-90\%), when working at $37 \mathrm{kHz}$ (320 $\mathrm{W})$ and $75^{\circ} \mathrm{C}$. Moreover, only Knoevenagal product 29 was obtained skipping US irradiation using piperidine, basic alumina and acidic alumina as a catalyst with a trace amount of the expected bicyclic derivative 28.

Scheme 23

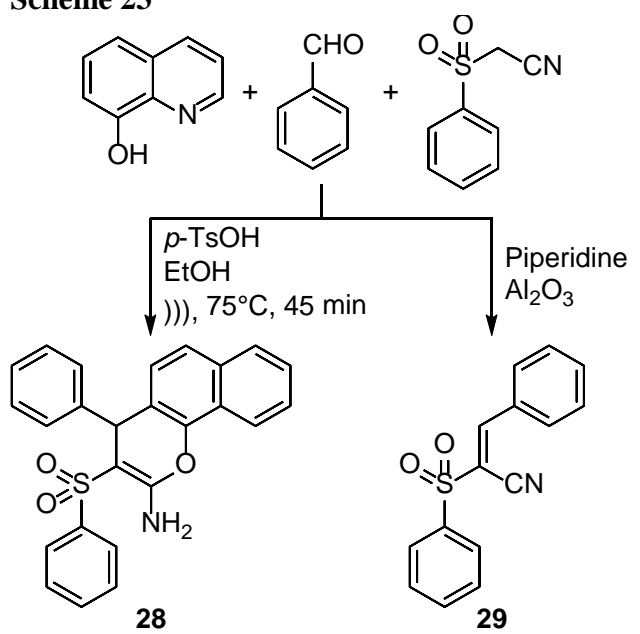

Catalyst-free MCRs for the condensation of malononitrile, 2-naphthol/resorcinol, various aromatic aldehydes, and ammonium acetate for the synthesis of a wide range of valuable dihydroquinoline derivatives $\mathbf{3 0}$ under US irradiation in aqueous media $(40 \mathrm{kHz}, 250 \mathrm{~W})$ at $60^{\circ} \mathrm{C}$ have been described by Pagadala et al. ${ }^{37}$ (Scheme 24). This heterogeneous sonochemical method is bestowed with several advantages, such as cost effectiveness, high yields (90-97\%) and short reaction times (60-90 min) while avoiding the use of a catalyst. Moreover, designing catalyst-free ultrasonic MCRs using water as a sole solvent is the best green chemistry choice because of the easier workup procedure and the inherent environmental advantages of such a process. 


\section{Scheme 24}<smiles>N#CCC(=O)ON</smiles><smiles>[R]c1ccc(C2C(C#N)=C(N)Nc3cc(O)ccc32)cc1</smiles>

30

2,3-Dihydroquinazolin-4(1H)-one derivatives are another important class of fused heterocyclic compounds that possess valuable biological and pharmacological activity. Their widespread application has led to an investigation into the synthesis of 2,3-dihydroquinazolin-4(1H)-one derivatives $\mathbf{3 1}$ by Chen et $\mathrm{al}^{38}$. The syntheses of these compounds were carried out under US irradiation in aqueous media at $40-42^{\circ} \mathrm{C}$ by the one-pot three-component condensation of isatoic anhydride, aromatic aldehyde, and amine using dodecylbenzenesulfonic acid (DBSA) serving at once as Brønsted acid catalyst and surfactant. Yields of $80-92 \%$ of 2,3-disubstituted 2,3-dihydroquinazolin-4(1H)-one derivatives 30 were achieved in only $1-$ $2 \mathrm{~h}$ under mild reaction conditions with US activation $(25-40 \mathrm{kHz}, 250 \mathrm{~W})$ with no need for organic solvents (Scheme 25). The same reactions required longer reaction times $(2-5 \mathrm{~h})$ and gave lower yields $(62-76 \%)$ under reflux without using US.

\section{Scheme 25}
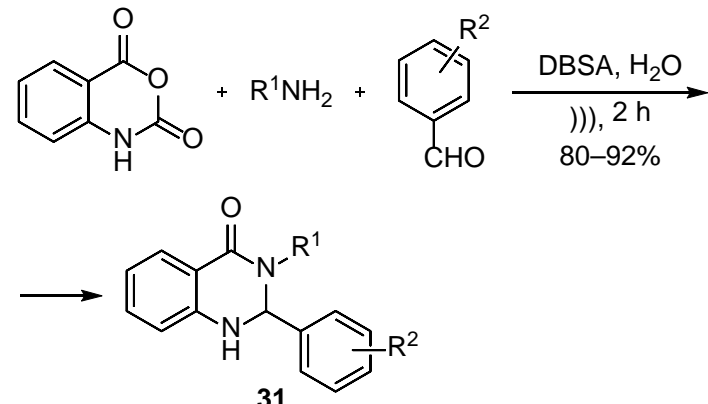

$\mathrm{R}^{1}=\mathrm{Et}, \mathrm{Me}, \mathrm{Ph} ; \mathrm{R}^{2}=\mathrm{H}, 4-\mathrm{Cl}, 4-\mathrm{NO}_{2}, 4-\mathrm{Me}$

Nikpassand et al. ${ }^{39}$ have described an environmentally benign US-assisted procedure for the synthesis of novel benzoxazole derivatives 32 using a number of different azo-linked salicylic acids and 2-amino-4-chlorophenols at room temperature (Scheme 26). US was an alternative energy source in this green process which gave benzoxazole derivatives 32 in short reaction times (10 min) at room temperature in $96 \%$ yield, as compared to $60 \%$ yield achieved without US at $80^{\circ} \mathrm{C}$ in ethanol under stirring for $17 \mathrm{~h}$.

\section{Scheme 26}

Ar<smiles>CN=Nc1ccc(O)c(C(=O)[OH+])c1</smiles>

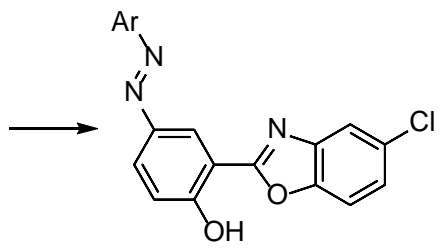

32

Naeimi et al. ${ }^{40}$ have presented a simple and efficient method for the one-pot synthesis of 2-arylbenzoxazoles 33a,b which were directly synthesized from $o$-aminophenol and aromatic or heteroaromatic aldehydes (Scheme 27). The reaction was catalyzed with KCN supported by multi-walled carbon nanotubes (MWCNT) in DMF under US irradiation. Substituted benzaldehydes in particular, with either electron-donating or -withdrawing groups were used to afford 2-arylbenzoxazoles 33a under US irradiation (20 kHz, 60W) in short reaction times (35 s), excellent yields (90-96\%) and high purity. A benzoin condensation mechanism for this synthesis was proposed: the adsorption of potassium ions on MWCNT leads to a nucleophilicity increase of cyanide ion, accelerating the formation of benzoin intermediate with the aldehyde. This 
intermediate may undergo a nucleophilic addition with 2-aminophenol that after an intramoleclar nucleophilic attack of the hydroxyl group affords the corresponding product 33a,b.

\section{Scheme 27}

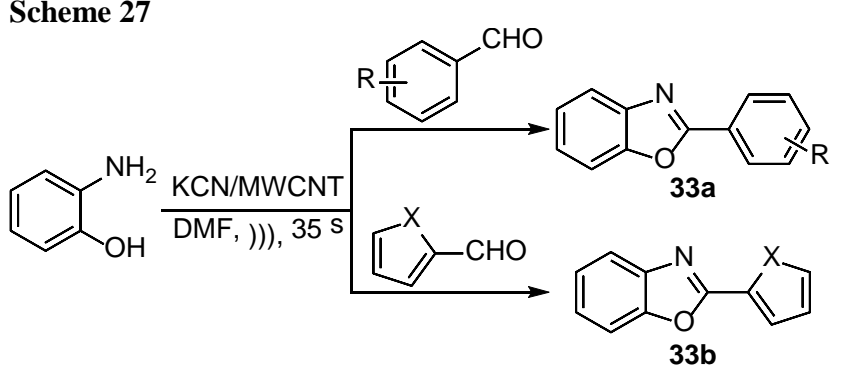

Chen, He et al. ${ }^{41}$ have developed a simple and efficient US-assisted synthesis for fluoro-containing heterocycles 35 via a $S_{\mathrm{N}} \mathrm{Ar}$ reaction of tetrafluoroterephthalonitrile with various nucleophiles 34 (Scheme 28). The reaction proceeded very quickly at room temperature under 5 -min sonication $(25 \mathrm{kHz}, 150 \mathrm{~W})$ to give heterocycles in moderate to good yields (50$80 \%)$. Moreover, compounds 34 can be used as good ligands in metal-organic frameworks thanks to the unique properties of the fluorine atoms.<smiles>N#Cc1c(F)c(F)c(C#N)c(F)c1F</smiles>

$$
\mathrm{X}=\mathrm{OH}, \mathrm{SH}, \mathrm{NH}_{2} ; \mathrm{Y}=\mathrm{O}, \mathrm{S}, \mathrm{NH}
$$

The sonochemical one-pot synthesis of benzo[f]indole-4,9-diones 36 from 1,4-naphthoquinone and $\alpha$-aminoacetals has recently been described in detail by Luu et al. ${ }^{42}$ (Scheme 29). The authors sought to develop an efficient synthesis for $p$ indolequinones by using the nucleophilicity of aminoquinones and a 1,4-naphthoquinone amination reaction followed by cyclization to the corresponding benzo-fused indolequinones. This method is a straightforward protocol for obtaining benzo[f]indole-4,9-diones 36 in high yields (77-88\%). The proposed mechanism includes the formation in situ of an aldehyde which undergoes a nucleophilic attack of aminoquinone under mild conditions.

\section{Scheme 29}

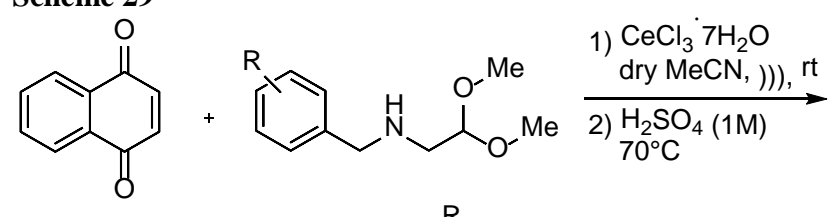<smiles>[R]c1ccc(Cn2ccc3c2C(=O)c2cc(CC)ccc2C3=O)cc1</smiles>

Reddy et al. ${ }^{43}$ have presented a straightforward and efficient protocol for the synthesis of pharmaceutically relevant $1,4-$ dihydropyrimido[1,2-a]benzimidazole-3-carboxylates 37 via the three-component reaction of an aldehyde, $1 H$ benzimidazol-2-amine, and an active methylene compound in the presence of di(n-butyl)ammonium chlorosulfonate ionic liquid (DBAIL) under US irradiation (30-60 min) (Scheme 30). They described the simple, green, and efficient one-pot, US-assisted synthesis of a variety of bioactive 1,4-dihydrobenzo[4,5]imidazo[1,2-a]pyrimidine-3-carboxylates (70-95\% yields) from easily available starting materials under solvent-free conditions. The US/IL combination clearly shows its potential for future broad contributions to green and sustainable chemistry. 


\section{Scheme 30}
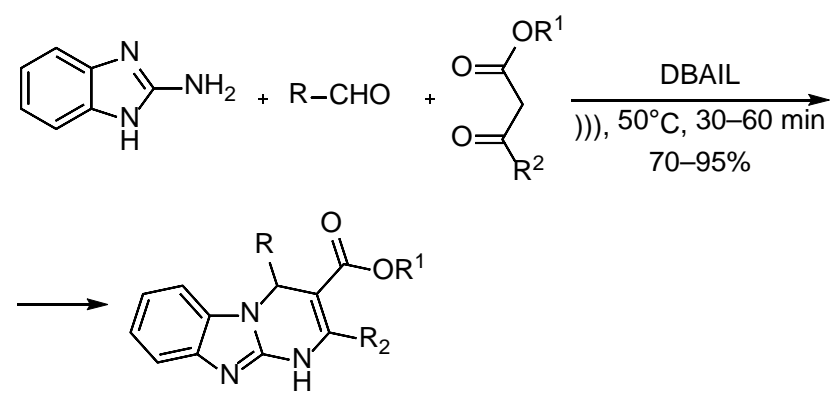

37

Nallapati et al. ${ }^{44}$ have reported the US-assisted derivatization of the antipsychotic drug skeleton olanzapine by linking its $\mathrm{N}$-10 position with suitable $N$-substituted 1,2,3-triazole moieties through a methylene linker giving products 38 (Scheme 31). Sonochemical CuAAC (US at $35 \mathrm{kHz}$ ) gave a series of more than twenty triazole derivatives 38 with different $N$ substituents in only 20-35 min at room temperature. The use of US provided cleaner and quicker access to these compounds (70-90\% yields) than the conventional thermal process. These compounds were evaluated for their potential of in vitro PDE4 inhibitory effect for the treatment of the positive, negative, or cognitive symptoms of schizophrenia.

\section{Scheme 31}<smiles>C#CCN1c2ccccc2N=C(N2CCN(C)CC2)c2cc(C)sc21</smiles><smiles>[R]n1cc(CN2c3cc(C(C)C)ccc3N=C(N3CCN(C)CC3)c3cc(C)sc32)nn1</smiles>

38

The US-promoted synthesis of novel fused heterocycles via criss-cross cycloaddition was reported by Safari et al. (2016). ${ }^{45}$ In particular, perhydrotriazolotriazoledithione derivatives 39 were synthesized via two sequential 1,3-dipolar cycloadditions under US irradiation $(24 \mathrm{kHz})$ from various azine derivatives and potassium isothiocyanate using glacial $\mathrm{AcOH}$ as the solvent (Scheme 32). The best advantages of the method are selectivity, mild reaction conditions (room temperature), short reaction times (10-35 min) and excellent yields (80-98\%).

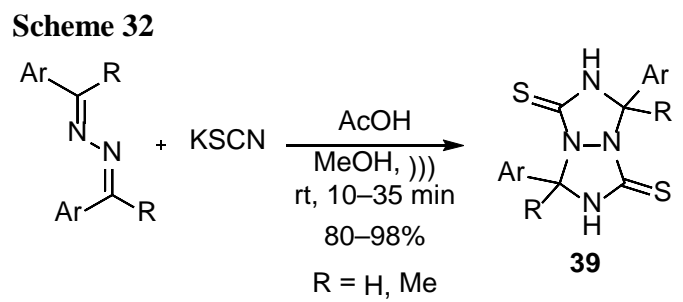

The recent impressive advances in the design of ultrasound- and ball mill-assisted synthetic protocols for the cyclization of heterocycles have shed light on the huge potential of the sonochemical and mechanochemical techniques. New heterocycle libraries for the pharmaceutical lead generation have been prepared under ultrasound treatment and ball milling. Besides higher yields, shorter reaction times, and greener procedures, higher selectivity and process intensification may pave the way for industrial applications.

\section{References}

1. Cravotto, G.; Tagliapietra, S.; Caporaso, M.; Garella, D. Borretto, E.; Di Stilo A. Chem. Heterocycl. Compd. 2013, 49, 811. [Khim. Geterotsikl. Soedin. 2013, 869.]

2. Cravotto, G.; Cintas, P. Chem. Sci. 2012, 3, 295.

3. Zbancioc, G.; Florea, O.; Jones, P.; Mangalagiu, I. I. Ultrason. Sonochem. 2012, 19, 399.

4. https://goldbook.iupac.org/MT07141.html

5. Stolle, A.; Szuppa, T.; Leonhardt, S. E. S.; Ondruschka, B. Chem. Soc. Rev. 2011, 40, 2317.

6. Kaupp, G.; Schmeyers, J.; Kuse, A.; Atfeh, A. Angew. Chem., Int. Ed. 1999, 38, 2896.

7. M'hamed, M. O. Synth. Commun. 2015, 45, 2511.

8. Akelis, L.; Rousseau, J.; Juskenas, R.; Dodonova, J.; Rousseau, C.; Menuel, S.; Prevost, D.; Tumkevičius, S.; Monflier, E.; Hapiot, F. Eur. J. Org. Chem. 2016, 31.

9. Miklós, F.; Petrisor, A.; Fülöp, F. ARKIVOC 2015, (vii), 158. 
10. Jin, M.; Song, G.; Li, Z.; Zhou, F.; Fan, B.; Ouyang, P. J. Heterocycl. Chem. 2014, 51, 1838.

11. Banerjee, M.; Chatterjee, A.; Kumar, V.; Bhutia, Z. T.; Khandare, D. G.; Majikb, M. S.; Roy. B. G. RSC Adv. $2014,4,39606$.

12. Tullberg, E.; Schacher, F.; Peters, D.; Frejd, T. Synthesis 2006, 1183.

13. Shy, H.; Mackin, P.; Orvieto, A. S.; Gharbharan, D.; Peterson, G. R.; Bampos, N.; Hamilton, T. D. Faraday Discuss. 2014, 170, 59.

14. Weiße, M.; Zille, M.; Jacob, K.; Schmidt, R.; Stolle, A. Chem.-Eur. J. 2015, 21, 6511.

15. Chen, X.; Jia, C.; Cao, L.; Zhang, D.; Liu, S. Chem. Res. Chin. Univ. 2015, 31, 543.

16. Wang, S.-F.; Guo, C.-L.; Cui, K.-K.; Zhu, Y.-T.; Ding, J.-X.; Zou, X.-Y.; Li, Y.-H. Ultrason. Sonochem. 2015, $26,81$.

17. Franco, M. S. F.; Casagrande, G. A.; Raminelli, C.; Moura, S.; Rossatto, M.; Quina, F. H.; Pereira, C. M. P.; Flores, A. F. C.; Pizzuti, L. Synth. Commun. 2015, 45, 692.

18. Mady, M. F.; Awad, G. E. A.; Jørgensen, K. B. Eur. J. Med. Chem. 2014, 84, 433.

19. Pagadala, R.; Maddila, S.; Jonnalagadda, S. B. Green Chem. Lett. Rev. 2014, 7, 131.

20. Wang, L.; Chen T.; Chen, S.-C.; Chen, Q.; He, M.-Y. J. Hetercycl. Chem. 2014, 51, 1536.

21. Carradori, S.; Secci, D.; D'Ascenzio, M.; Chimenti, P.; Bolasco A. J. Heterocycl. Chem. 2014, 51, 1856.

22. Mantu, D.; Maftei, D.; Iurea D. Med. Chem. Res. 2014, 23, 2909.

23. Nemati, F.; Nikkhah, S. H.; Elhampour, A. Chinese Chem. Lett. 2015, 26, 1397.

24. Zarganes-Tzitzikas, T.; Chandgude, A. L.; Doemling, A. Chem. Rec. 2015, 15, 981.

25. Elders, N.; Van der Born, D.; Hendrickx, L. J. D.; Timmer, B. J. J.; Krause, A.; Janssen, E.; de Kanter, F. J. J.; Ruijter, E.; Orru, R. V. A. Angew. Chem., Int. Ed. 2009, 48, 5856.

26. Shabalala, N. G.; Pagadala, R.; Jonnalagadda, S. B. Ultrason. Sonochem. 2015, 27, 423.

27. Mady, M. F.; Awad, G. E. A.; Jørgensen, K. B. Eur. J. Med. Chem. 2014, 84, 433.

28. Rezki, N. Molecules 2016, 21, 505.

29. Naeimi, H.; Kiani F. Ultrason. Sonochem. 2015, 27, 408.

30. Carradori, S.; Secci, D.; D'Ascenzio, M.; Chimenti, P.; Bolasco A. J. Heterocyclic Chem. 2014, 51, 1856.

31. Bhosle, M. R.; Khillare, L. D.; Dhumal, S. T.; Mane, R. A. Lett. Org. Chem., 2016, 13, 148.

32. Darehkordi, A.; Ghazi, S. Arab. J. Chem. 2015, http://dx.doi.org/10.1016/j.arabjc.2015.01.010.

33. Mantu, D.; Maftei, D.; Iurea D. Med. Chem. Res. 2014, 23, 2909.

34. Gouvêa, D.P.; Berwaldt, G. A.; Neuenfeldt, P. D.; Nunes, R. J.; Almeidac, W. P.; Cunico. W. J. Braz. Chem. Soc. 2016, $27,1109$.

35. Tabassum, S.; Govindaraju, S.; Khan, R.-R.; Pasha M. A. Ultrason. Sonochem. 2015, 24, 1.

36. Al-Bogami, A. S.; Saleh, T. S.; Zayed, E. M. Ultrason. Sonochem. 2013, $20,1194$.

37. Pagadala, R.; Maddila, S.; Jonnalagadda, S. B. Green Chem. Lett. Rev. 2014, 7, 131.

38. Chen, B.-H.; Li, J.-T.; Chen, G.-F. Ultrason. Sonochem. 2015, 23, 59.

39. Nikpassand, M.; Fekri, L. Z.; Farokhian, P. Ultrason. Sonochem. 2016, $28,341$.

40. Naeimi, H.; Rahmatinejad, S.; Nazifi, Z. S. J. Taiwan Inst. Chem. Eng. 2016, 58, 1.

41. Wang, L.; Chen T.; Chen, S.-C.; Chen, Q.; He, M.-Y. J. Hetercycl. Chem. 2014, 51, 1536.

42. Luu, Q. H.; Guerra, J. D.; Castañeda, C. M.; Martinez, M. A.; Saunders, J.; Garcia, B. A.; Gonzales, B. V.; Aidunuthula, A. R.; Mito, S. Tetrahedron Lett., 2016, 57, 2253.

43. Reddy, M. V.; Reddy, A. V. S.; Jeong, Y. T. Res. Chem. Intermed. 2016, 42, 4893.

44. Nallapati, S. B.; Sreenivas, B. Y.; Bankala, R.; Parsa, K. V. L.; Sripelly, S.; Mukkantib, K.; Pal, M. RSC Adv. 2015, $5,94623$.

45. Safari, J.; Gandomi-Ravandi, S.; Ghotbinejad, M. J. Saudi Chem. Soc. 2016, 20, 20. 